\title{
Dissipative Properties of $\omega$-Order Preserving Partial Contraction Mapping in Semigroup of Linear Operator
}

\author{
Akinola Yussuff Akinyele, Kamilu Rauf*, Aasa Moses Adebowale, Omosowon Jude Babatunde \\ Department of Mathematics, University of Ilorin, Ilorin, Nigeria \\ Email: olaakinyele04@mail, *krauf@unilorin.edu.ng, aasamoses@y aoo.com, omosow onjude@yahoo.com
}

How to cite this paper: Akinyele, A.Y, Rauf, K., Adebowale, A.M. and Babatunde, O.J. (2019) Dissipative Properties of $\omega$-Order Preserving Partial Contraction Mapping in Semigroup of Linear Operator. Advances in Pure Mathematics, 9, 544-550. https://doi.org/10.4236/apm.2019.96026

Received: March 5, 2019

Accepted: June 25, 2019

Published: June 28, 2019

Copyright ( 2019 by author(s) and Scientific Research Publishing Inc. This work is licensed under the Creative Commons Attribution International License (CC BY 4.0).

http://creativecommons.org/licenses/by/4.0/

\begin{abstract}
This paper consists of dissipative properties and results of dissipation on infinitesimal generator of a $C_{0}$-semigroup of $\omega$-order preserving partial contraction mapping $\left(\omega-O C P_{n}\right)$ in semigroup of linear operator. The purpose of this paper is to establish some dissipative properties on $\omega$ - $O C P_{n}$ which have been obtained in the various theorems (research results) and were proved.
\end{abstract}

\section{Keywords}

Semigroup, Linear Operator, Dissipative Operator, Contraction Mapping and Resolvent

\section{Introduction}

Suppose $X$ is a Banach space, $X_{n} \subseteq X$ a finite set, $(T(t))_{t \geq 0}$ the $C_{0}$-semigroup that is strongly continuous one-parameter semigroup of bounded linear operator in $X$. Let $\omega$ - $O C P_{n}$ be $\omega$-order-preserving partial contraction mapping (semigroup of linear operator) which is an example of $C_{0}$-semigroup. Furthermore, let $M m(\mathbb{N})$ be a matrix, $L(X)$ a bounded linear operator on $X$, $P_{n}$ a partial transformation semigroup, $\rho(A)$ a resolvent set, $F(x)$ a duality mapping on $X$ and $A$ is a generator of $C_{0}$-semigroup. Taking the importance of the dissipative operator in a semigroup of linear operators into cognizance, dissipative properties characterized the generator of a semigroup of linear operator which does not require the explicit knowledge of the resolvent.

This paper will focus on results of dissipative operator on $\omega-O C P_{n}$ on Banach space as an example of a semigroup of linear operator called $C_{0}$-semigroup.

Yosida [1] proved some results on differentiability and representation of one-parameter semigroup of linear operators. Miyadera [2], generated some 
strongly continuous semigroups of operators. Feller [3], also obtained an unbounded semigroup of bounded linear operators. Balakrishnan [4] introduced fractional powers of closed operators and semigroups generated by them. Lumer and Phillips [5], established dissipative operators in a Banach space and Hille \& Philips [6] emphasized the theory required in the inclusion of an elaborate introduction to modern functional analysis with special emphasis on functional theory in Banach spaces and algebras. Batty [7] obtained asymptotic behaviour of semigroup of operator in Banach space. More relevant work and results on dissipative properties of $\omega$-Order preserving partial contraction mapping in semigroup of linear operator could be seen in Engel and Nagel [8], Vrabie [9], Laradji and Umar [10], Rauf and Akinyele [11] and Rauf et al. [12].

\section{Preliminaries}

Definition 2.1 ( $C_{0}$-Semigroup) [9]

$C_{0}$-Semigroup is a strongly continuous one parameter semigroup of bounded linear operator on Banach space.

Definition $2.2\left(\omega-O C P_{n}\right)[11]$

Transformation $\alpha \in P_{n}$ is called $\omega$-order-preserving partial contraction mapping if $\forall x, y \in \operatorname{Dom} \alpha: x \leq y \Rightarrow \alpha x \leq \alpha y$ and at least one of its transformation must satisfy $\alpha y=y$ such that $T(t+s)=T(t) T(s)$ whenever $t, s>0$ and otherwise for $T(0)=I$.

Definition 2.3 (Subspace Semigroup) [8]

A subspace semigroup is the part of $A$ in $Y$ which is the operator $A_{*}$ defined by $A_{*} y=A y$ with domain $D\left(A_{*}\right)=\{y \in D(A) \cap Y: A y \in Y\}$.

Definition 2.4 (Duality set)

Let $X$ be a Banach space, for every $x \in X$, a nonempty set defined by $F(x)=\left\{x^{*} \in X^{*}:\left(x, x^{*}\right)=\|x\|^{2}=\left\|x^{*}\right\|^{2}\right\}$ is called the duality set.

Definition 2.5 (Dissipative) [9]

A linear operator $(A, D(A))$ is dissipative if each $x \in X$, there exists $x^{*} \in F(x)$ such that $\operatorname{Re}\left(A x, x^{*}\right) \leq 0$.

\subsection{Properties of Dissipative Operator}

For dissipative operator $A: D(A) \subseteq X \rightarrow X$, the following properties hold:

a) $\lambda-A$ is injective for all $\lambda>0$ and

$$
\left\|(\lambda-A)^{-1}\right\| \leq 1 / \lambda\|y\|
$$

for all $y$ in the range $\operatorname{rg}(\lambda-A)=(\lambda-A) D(A)$.

b) $\lambda-A$ is surjective for some $\lambda>0$ if and only if it is surjective for each $\lambda>0$. In that case, we have $(0, \infty) \subset \rho(A)$, where $\rho(A)$ is the resolvent of the generator $A$.

c) $A$ is closed if and only if the range $\operatorname{rg}(\lambda-A)$ is closed for some $\lambda>0$.

d) If $\operatorname{rg}(A) \subseteq D(A)$, that is if $A$ is densely defined, then $A$ is closable. its closure $A$ is again dissipative and satisfies $\operatorname{rg}(\lambda-A)=\operatorname{rg}(\lambda-A)$ for all $\lambda>0$. 


\section{Example 1}

$2 \times 2$ matrix $\left[M_{m}(\mathbb{N} \cup\{0\})\right]$

Suppose

$$
A=\left(\begin{array}{ll}
1 & 2 \\
2 & 2
\end{array}\right)
$$

and let $T(t)=e^{t A}$, then

$$
e^{t A}=\left(\begin{array}{cc}
e^{t} & e^{2 t} \\
e^{2 t} & e^{2 t}
\end{array}\right)
$$

$3 \times 3$ matrix $\left[M_{m}(\mathbb{N} \cup\{0\})\right]$

Suppose

$$
A=\left(\begin{array}{lll}
1 & 2 & 3 \\
1 & 2 & 2 \\
- & 2 & 3
\end{array}\right)
$$

and let $T(t)=e^{t A}$, then

$$
e^{t A}=\left(\begin{array}{lll}
e^{t} & e^{2 t} & e^{3 t} \\
e^{t} & e^{2 t} & e^{2 t} \\
I & e^{2 t} & e^{3 t}
\end{array}\right)
$$

\section{Example 2}

In any $2 \times 2$ matrix $\left[M_{m}(\mathbb{C})\right]$, and for each $\lambda>0$ such that $\lambda \in \rho(A)$ where $\rho(A)$ is a resolvent set on $X$.

Also, suppose

$$
A=\left(\begin{array}{ll}
1 & 2 \\
- & 2
\end{array}\right)
$$

and let $T(t)=e^{t A_{\lambda}}$, then

$$
e^{t A_{\lambda}}=\left(\begin{array}{cc}
e^{t \lambda} & e^{2 t \lambda} \\
I & e^{2 t \lambda}
\end{array}\right)
$$

\section{Example 3}

Let $X=C_{u b}(\mathbb{N} \cup\{0\})$ be the space of all bounded and uniformly continuous function from $\mathbb{N} \cup\{0\}$ to $\mathbb{R}$, endowed with the sup-norm $\|\cdot\|_{\infty}$ and let $\{T(t) ; t \geq 0\} \subseteq L(X)$ be defined by

$$
[T(t) f](s)=f(t+s)
$$

For each $f \in X$ and each $t, s \in \mathbb{R}_{+}$, it is easily verified that $\{T(t) ; t \geq 0\}$ satisfies Examples 1 and 2 above.

\section{Example 4}

Let $X=C[0,1]$ and consider the operator $A f=-f^{\prime}$ with domain $D(A)=\left\{f \in C^{\prime}[0,1]: f(0)=0\right\}$. It is a closed operator whose domain is not dense. However, it is dissipative, since its resolvent can be computed explicitly as

$$
R(\lambda, A) f(t)=\int_{0}^{t} e^{-\lambda(t-s)} f(s) \mathrm{d} s
$$


for $t \in[0,1], f \in C[0,1]$. Moreover, $\|R(\lambda, A)\| \leq \frac{1}{\lambda}$ for all $\lambda>0$. Therefore $(A, D(A))$ is dissipative.

\subsection{Theorem (Hille-Yoshida [9])}

A linear operator $A: D(A) \subseteq X \rightarrow X$ is the infinitesimal generator for a $C_{0}$-semigroup of contraction if and only if

1) $A$ is densely defined and closed,

2) $(0,+\infty) \subseteq \rho(A)$ and for each $\lambda>0$

$$
\|R(\lambda, A)\|_{L(X)} \leq \frac{1}{\lambda}
$$

\subsection{Theorem (Lumer-Phillips [5])}

Let $X$ be a real, or complex Banach space with norm $\|\cdot\|$, and let us recall that the duality mapping $F: X \rightarrow 2^{x}$ is defined by

$$
F(x)=\left\{x^{*} \in X^{*} ;\left(x, x^{*}\right)=\|x\|^{2}=\left\|x^{*}\right\|^{2}\right\}
$$

for each $x \in X$. In view of Hahn-Banach theorem, it follows that, for each $x \in X, F(x)$ is nonempty.

\subsection{Theorem (Hahn-Banach Theorem [2])}

Let $V$ be a real vector space. Suppose $p: V \in[0,+\infty]$ is mapping satisfying the following conditions:

1) $p(0)=0$;

2) $p(t x)=t p(x)$ for all $x \in V$ and real of $t \geq 0$; and

3) $p(x+y) \leq p(x)+p(y)$ for every $x, y \in v$.

Assume, furthermore that for each $x \in V$, either both $p(x)$ and $p(-x)$ are $\infty$ or that both are finite.

\section{Main Results}

In this section, dissipative results on $\omega-O C P_{n}$ as a semigroup of linear operator were established and the research results(Theorems) were given and proved appropriately:

\section{Theorem 3.1}

Let $A \in w-O C P_{n}$ where $A: D(A) \subseteq X \rightarrow X$ is a dissipative operator on a Banach space $X$ such that $\lambda-A$ is surjective for some $\lambda>0$. Then

1) the part $A$, of $A$ in the subspace $X_{0}=\overline{D(A)}$ is densely defined and generates a constrain semigroup in $X_{0}$, and

2) considering $X$ to be a reflexive, $A$ is densely defined and generates a contraction semigroup.

\section{Proof}

We recall from Definition 2.3 that

$$
A_{*} x=A x
$$


for

$$
x \in D\left(A_{*}\right)=\left\{x \in x \in D(A): A x \in X_{0}\right\}=R(\lambda, A) X_{0}
$$

Since $R(\lambda, A)$ exists for $\lambda>0$, this implies that $R(\lambda, A)_{*}=R\left(\lambda, A_{*}\right)$, hence

$$
(0, \infty) \subset \rho\left(A_{*}\right)
$$

we need to show that $D\left(A_{*}\right)$ is dense in $X_{0}$.

Take $x \in D(A)$ and set $x_{n}=n R(n, A) x$. Then $x_{n} \in D(A)$ and

$$
\lim _{n \rightarrow \infty} x_{n}=\lim _{n \rightarrow \infty} R(n, A) A x+x=x,
$$

since $\|R(n, A)\| \leq \frac{1}{n}$. Therefore the operators $n R(n, A)$ converge pointwise on $D(A)$ to the identity. Since $\|n R(n, A)\| \leq 1$ for all $n \in \mathbb{N}$, we obtain the convergence of $y_{n}=n R(n, A) y \rightarrow y$ for all $y \in X_{0}$. If for each $y_{n}$ in $D\left(A_{*}\right)$, the density of $D\left(A_{*}\right)$ in $X_{0}$ is shown which proved (i).

To prove (ii), we need to obtain the density of $D(A)$.

Let $x \in X$ and define $x_{n}=n R(n, A) x \in D(A)$. The element $y=n R(1, A) x$, also belongs to $D(A)$. Moreover, by the proof of (i) the operators $n R(n, A)$ converges towards the identity pointwise on $X_{0}=\overline{D(A)}$. It follows that

$$
y_{n}=R(1, A) x_{n}=n R(n, A) R(1, A) x \rightarrow y \text { for } n \rightarrow \infty
$$

Since $X$ is reflexive and $\left\{x_{n}: n \in \mathbb{N}\right\}$ is bounded, there exists a subsequence, still denoted by $\left(x_{n}\right)_{(n \in \mathbb{N})}$, that converges weakly to some $z \in X$. Since $x_{n} \in D(A)$, implies that $z \in \overline{D(A)}$.

On the other hand, the elements $x_{n}=(1-A) y_{n}$ converges weakly to $z$, so the weak closedness of $A$ implies that $y \in D(A)$ and $x=(1-A) y=z \in \overline{D(A)}$ which proved (ii).

Theorem 3.2

The linear operator $A: D(A) \subseteq X \rightarrow X$ is a dissipative if and only if for each $x \in D(A)$ and $\lambda>0$, where $A \in \omega-O C P_{n}$, then we have

$$
\left\|\left(\lambda_{1}-A\right) x\right\| \geq \lambda\|x\|
$$

\section{Proof}

Suppose $A$ is dissipative, then, for each $x \in D(A)$ and $\lambda>0$, there exists $x^{*} \in F(x)$ such that $\operatorname{Re}\left(\lambda x-A x, x^{*}\right) \leq 0$. Therefore

$$
\|x\|\|\lambda x-A x\| \geq|(\lambda x-A x, x)| \geq \operatorname{Re}(\lambda x-A x, x) \geq \lambda\|x\|^{2}
$$

and this completes the proof. Next, let $x \in D(A)$ and $\lambda>0$.

Let $y_{\lambda}^{*} \in F(\lambda x-A x)$ and let us observe that, by virtue of (3.3), $\lambda x-A x=0$ $\Rightarrow \quad x=0$.

So, in this case, we clearly have $\operatorname{Re}\left(x^{*}, \lambda x-A x\right)=0$. Therefore, by assuming that $\lambda x-A x \neq 0$. As a consequence, $y_{\lambda}^{*} \neq 0$, and thus

$$
z_{\lambda}^{*}=\frac{y_{\lambda}^{*}}{\left\|y_{\lambda}^{*}\right\|}
$$


lies on the unit ball, i.e. $\left\|z_{\lambda}^{*}\right\|=1$. We have $\left(\lambda x-A x, z_{\lambda}^{*}\right)=\|\lambda x-A x\| \geq \lambda\|x\| \Rightarrow$ $\operatorname{Re}\left(x, z_{\lambda}^{*}\right)-\operatorname{Re}\left(A x, z_{\lambda}^{*}\right) \leq \lambda\|x\|-\operatorname{Re}\left(A x, z_{\lambda}^{*}\right)$ hence

$$
\operatorname{Re}\left(A x, z_{\lambda}^{*}\right) \leq 0
$$

and $\operatorname{Re}\left(z_{\lambda}^{*}, x\right) \geq\|x\|-\frac{1}{\lambda}\|A x\|$. Now, let us recall that the closed unit ball in $X^{*}$ is weakly-star compact. Thus, the net $\left(z_{\lambda}^{*}\right)_{\lambda>0}$ has at least one weak-star cluster point $z^{*} \in X^{*}$ with

$$
\left\|z^{*}\right\| \leq 1
$$

From (3.4), it follows that $\operatorname{Re}\left(A x, z^{*}\right) \leq 0$ and $\operatorname{Re}\left(x, z^{*}\right) \geq\|x\|$. Since $\operatorname{Re}\left(x, z^{*}\right) \leq\left|\left(x, z^{*}\right)\right| \leq\|x\|$, it follows that $\left(x, z^{*}\right)=\|x\|$. Hence $x^{*}=\|x\| z^{*} \in F(x)$ and $\operatorname{Re}\left(A x, x^{*}\right) \leq 0$ and this completes the proof.

\section{Proposition 3.3}

Let $A: D(A) \subseteq X \rightarrow X$ be infinitesimal generator of a $C_{0}$-semigroup of contraction and $A \in \omega-O C P_{n}$. Suppose $X_{*}=D(A)$ is endowed with the graph-norm $|\cdot|_{D(A)}: X_{*} \rightarrow \mathbb{N} \bigcup\{0\}$ defined by $|u|_{D(A)}=\|u-A u\|$ for $u \in X_{*}$. Then operator $A_{*}: D\left(A_{*}\right) \subseteq X_{*} \rightarrow X_{*}$ defined by

$$
\left\{\begin{array}{l}
D\left(A_{*}\right)=\left\{x \in X_{*} ; A x \in X_{*}\right\} \\
A_{*} x=A x, \text { for } x \in D\left(X_{*}\right)
\end{array}\right.
$$

is the infinitesimal generator of a $C_{0}$-semigroup of contractions on $X_{*}$.

\section{Proof}

Let $\lambda>0$ and $f \in X_{*}$ and let us consider the equation $\lambda u-A u=F$ Since $A$ generates a $C_{0}$-semigroup of contraction [6], it follows that this equation has a unique solution $u \in D(A)$.

Since $f \in X_{*}$, we conclude that $A u \in D(A)$ and thus $u \in D\left(A_{*}\right)$.

Thus $\lambda u-A_{*} u=f$. On the other hand, we have

$$
\begin{aligned}
& \left|\left(\lambda I-A_{*}\right)^{-1} f\right|_{D(A)}=\left\|(I-A)(\lambda I-A)^{-1} f\right\| \\
& =\left\|(\lambda I-A)^{-1}(I-A) f\right\| \leq \frac{1}{\lambda}\|f-A f\|=\frac{1}{\lambda}|f|_{D(A)}
\end{aligned}
$$

which shows that $A_{*}$ satisfies condition (ii) in Theorem 2.2. Moreover, it follows that $A_{*}$ is closed in $X_{*}$.

Indeed, as $(\lambda I-A)^{-1} \in L\left(X_{*}\right)$, it is closed, and consequently $\lambda I-A_{*}$ enjoys the same property which proves that $A_{*}$ is closed.

Now, let $x \in X_{*}, \lambda>0, A \in \omega-O C P_{n}$ and let $x_{\lambda}=\lambda x-A_{*} x$. Clearly $x_{\lambda} \in D\left(A_{*}\right)$, and in addition $\lim _{\lambda \rightarrow \infty}\left|x_{\lambda}-x\right|_{D(A)}=0$ Thus, $D\left(A_{*}\right)$ is dense in $X_{*}$ by virtue of Theorem 2.2, $A_{*}$ generates a $C_{0}$-semigroup of contraction on $X_{*}$. Hence the proof.

\section{Conclusion}

In this paper, it has been established that $\omega-O C P_{n}$ possesses the properties of dissipative operators as a semigroup of linear operator, and obtaining some dis- 
sipative results on $\omega-O C P_{n}$.

\section{Conflicts of Interest}

The authors declare no conflicts of interest regarding the publication of this paper.

\section{References}

[1] Yosida, K. (1948) On the Differentiability and Representation of One-Parameter Semigroups of Linear Operators. Journal of the Mathematical Society of Japan, 1, 15-21. https://doi.org/10.2969/jmsj/00110015

[2] Miyadera, I. (1952) Generation of Strongly Continuous Semigroups Operators. Tohoku Mathematical Journal, 4, 109-114. https://doi.org/10.2748/tmj/1178245412

[3] Feller, W. (1953) On the Generation of Unbounded Semigroup of Bounded Linear Operators. Annals of Mathematics, 58, 166-174. https://doi.org/10.2307/1969826

[4] Balakrishnan, A.V. (1960) Fractional Powers of Closed Operators and Semigroups Generated by Them. Pacific Journal of Mathematics, 10, 419-437. https://doi.org/10.2140/pjm.1960.10.419

[5] Lumer, G. and Phillips, R.S. (1961) Dissipative Operators in a Banach Space. Pacific Journal of Mathematics, 11, 679-698. https://doi.org/10.2140/pjm.1961.11.679

[6] Hille, E. and Phillips, R.S. (1981) Functional Analysis and Semigroups. American Mathematical Society, Providence, Colloquium Publications Vol. 31.

[7] Batty, C.J.K. (1994) Asymptotic Behaviour of Semigroup of Operators. Banach Center Publications, 30, 35-52. https://doi.org/10.4064/-30-1-35-52

[8] Engel, K. and Nagel, R. (1999) One-Parameter Semigroup for Linear Evolution Equations. Graduate Texts in Mathematics Vol. 194, Springer, New York.

[9] Vrabie, I.I. (2003) $\mathrm{C}_{0}$-Semigroup and Application. Mathematics Studies Vol. 191, Elsevier, North-Holland.

[10] Laradji, A. and Umar, A. (2004) Combinatorial Results for Semigroups of Order Preserving Partial Transformations. Journal of Algebra, 278, 342-359. https://doi.org/10.1016/j.jalgebra.2003.10.023

[11] Rauf, K. and Akinyele, A.Y. (2019) Properties of $\omega$-Order-Preserving Partial Contraction Mapping and Its Relation to $\mathrm{C}_{0}$-Semigroup. International Journal of Mathematics and Computer Science, 14, 61-68.

[12] Rauf, K., Akinyele, A.Y., Etuk, M.O., Zubair, R.O. and Aasa, M.A. (2019) Some Results of Stability and Spectra Properties on Semigroupn of Linear Operator. Advances of Pure Mathematics, 9, 43-51. https://doi.org/10.4236/apm.2019.91003 